\title{
Hypergeometric Functions with Integral Parameter Differences
}

\section{Karlsson, Per W.}

\section{Published in:}

Journal of Mathematical Physics

Link to article, DOI:

$10.1063 / 1.1665587$

Publication date:

1971

\section{Document Version}

Publisher's PDF, also known as Version of record

Link back to DTU Orbit

Citation (APA):

Karlsson, P. W. (1971). Hypergeometric Functions with Integral Parameter Differences. Journal of Mathematical Physics, 12(2), 270-271. https://doi.org/10.1063/1.1665587

\section{General rights}

Copyright and moral rights for the publications made accessible in the public portal are retained by the authors and/or other copyright owners and it is a condition of accessing publications that users recognise and abide by the legal requirements associated with these rights.

- Users may download and print one copy of any publication from the public portal for the purpose of private study or research.

- You may not further distribute the material or use it for any profit-making activity or commercial gain

- You may freely distribute the URL identifying the publication in the public portal

If you believe that this document breaches copyright please contact us providing details, and we will remove access to the work immediately and investigate your claim. 
1 We use the signature $(-,+,+,+)$ :

$$
\begin{aligned}
\Gamma_{\mu v}^{\sigma} & \equiv \frac{1}{2} g^{\sigma \tau}\left(g_{\mu \tau, v}+g_{v \tau, \mu}-g_{\mu v, \tau}\right), \\
R_{\mu v \sigma}{ }^{\tau} & \equiv \Gamma_{v \sigma, \mu}-\Gamma_{\mu \sigma, \tau}^{\tau}+\Gamma_{v \sigma} \rho \Gamma_{\mu \rho}{ }^{\tau}-\Gamma_{\mu \sigma} \rho \Gamma_{v \rho}{ }^{\tau}, \\
R_{\mu v} & \equiv R_{\mu \tau v}{ }^{\tau} .
\end{aligned}
$$

A comma denotes ordinary differentiation; a semicolon denotes covariant differentiation; $16 \pi G=1, c=1, G$ is the gravitational constant, and $c$ is the velocity of light. All other notation is defined in the text or is standard.

${ }^{2}$ For a review of the geometric theory, see Gravitation: An Introduction to Current Research, edited by L. Witten (Wiley, New York, 1962), Chap. 9.

3 J. L. Synge, Relativity: The General Theory (Interscience, New York, 1960), Chap. VIII; K. S. Thorne, Ph.D. thesis, University Microfilms, Ann Arbor, 1965.
4 The general solutions to Cases I and II of the possible field configurations and a particular solution of Case III is given in Ref. 2 and also in a paper by L. Witten, Colloq. Theor. Relativity, Centre Belge Rech. Math., Univ. (Louvain, Belgium), p. 59, 1960.

${ }_{5}$ This differs slightly from the line element given in Ref. 4. $\mu \equiv 0$ requires that we take the limit $l \rightarrow \infty$ in the line elements of Ref. 4 . The necessity that $l$ approach $\infty$ can be argued for on a variety of grounds, which we shall not present here.

${ }^{6}$ L. Marder, Proc. Roy. Soc. (London) A244, 524 (1958).

${ }_{7}^{7}$ M. A. Melvin, Phys. Letters 8, 65 (1964).

${ }^{8}$ M. A. Melvin, Phys. Rev. 139B, 225 (1965).

K. S. Thorne, Phys. Rev. 138B, 251 (1965).

${ }^{10}$ K. S. Thorne, Phys. Rev. 139B, 244 (1965).

11 J. L. Safko, Ann. Phys. (N.Y.) 58, 352 (1970).

${ }^{12}$ M. A. Melvin and J. S. Wallingford, J. Math. Phys. 7, 333 (1966).

${ }^{13}$ J. L. Safko, Bull. Am. Phys. Soc. 13, 1725 (1968).

14 J. L. Safko, Phys. Letters 28A, 347 (1968).

JOURNAL OF MATHEMATICAL PHYSICS VOLUME 12, NUMBER 2 FEBRARY 1971

\title{
Hypergeometric Functions with Integral Parameter Differences
}

Per W. Karlsson

Department of Physics, The Technical University, Lyngby, Denmark

(Received 20 July 1970)

\begin{abstract}
For a generalized hypergeometric function ${ }_{p} F_{q}(z)$ with positive integral differences between certain numerator and denominator parameters, a formula expressing the ${ }_{p} F_{o}(z)$ as a finite sum of lower-order functions is proved. From this formula, Minton's two summation theorems for $p=q+1, z=1$ are deduced, one of these under less restrictive conditions than assumed by Minton.
\end{abstract}

This paper deals with generalized hypergeometric functions ${ }_{p} F_{q}\left(a_{1}, \cdots, a_{p} ; b_{1}, \cdots, b_{q} ; z\right)$ having the special property that, with suitable enumeration of parameters, $a_{i}=b_{i}+m_{i}, i=1,2, \cdots, n$, where $m_{1}, \cdots, m_{n}$ are positive integers and $n \leq \min (p, q)$. It is assumed that $p \leq q+1$ and that no denominator parameter $b$ is a negative integer or zero. A function of this type may be expressed as a finite sum of ${ }_{p-n} F_{q \sim n}$ functions in the following way:

$\begin{aligned}{ }_{p} F_{q}\left[\begin{array}{r}b_{1}+m_{1}, \cdots, b_{n}+m_{n}, a_{n+1}, \cdots, a_{p} ; z \\ b_{1}, \cdots, b_{n}, b_{n+1}, \cdots,\end{array}\right] & \left.b_{q}\right]^{m_{1}} \ldots \sum_{m_{n}}^{m_{n}} A\left(j_{1}, \cdots, j_{n}\right) z^{J_{n}}{ }_{p-n} F_{q-n}\left[\begin{array}{c}a_{n+1}+J_{n}, \cdots, a_{v}+J_{n} ; z \\ b_{n+1}+J_{n}, \cdots, b_{q}+J_{n}\end{array}\right],\end{aligned}$

where

$$
J_{n}=j_{1}+\cdots+j_{n},
$$

$$
A\left(j_{1}, \cdots, j_{n}\right)=\left(\begin{array}{c}
m_{1} \\
j_{1}
\end{array}\right) \cdots\left(\begin{array}{c}
m_{n} \\
j_{n}
\end{array}\right) \frac{\left(b_{2}+m_{2}\right)_{J_{1}}\left(b_{3}+m_{3}\right)_{J_{2}} \cdots\left(b_{n}+m_{n}\right)_{J_{n-1}}\left(a_{n+1}\right)_{J_{n}} \cdots\left(a_{p}\right)_{J_{n}}}{\left(b_{1}\right)_{J_{1}}\left(b_{2}\right)_{J_{2}} \cdots\left(b_{n}\right)_{J_{n}}\left(b_{n+1}\right)_{J_{n}} \cdots\left(b_{q}\right)_{J_{n}}} \text {, }
$$

and

$$
(c)_{r}=\Gamma(c+r) / \Gamma(c) \text {. }
$$

By the principle of analytical continuation, Eq. (1) is valid whenever the functions involved are all analytic; restrictions upon the parameters imposed in the proof may thus be removed.

The proof is based upon an Eulerian integral representation given by Erdélyi, ${ }^{1}$ viz.,

$$
{ }_{p} F_{q}\left(a_{1}, \cdots, a_{p} ; b_{1}, \cdots, b_{q} ; z\right)=\frac{\Gamma\left(b_{1}\right) \Gamma\left(1-b_{1}+a_{1}\right)}{\Gamma\left(a_{1}\right) \exp \left(i \pi\left(b_{1}-a_{1}\right)\right)} \frac{i}{2 \pi} \times \int_{0}^{(1+)}(1-t)^{b_{1}-a_{1}-1} f(t) d t
$$

where

$$
f(t)=t_{p-1}^{a_{1}-1} F_{q-1}\left(a_{2}, \cdots, a_{p} ; b_{2}, \cdots, b_{q} ; z t\right),
$$


valid when $\operatorname{Re} a_{1}>0, b_{1}$ is not a negative integer or zero, and $|\arg (1-z)|<\pi$ if $p=q+1$. Now, as $a_{1}=b_{1}+m_{1}$, the branch point of the integrand at $t=1$ disappears, and the integral takes the form $\int_{C} f(t) d t /(t-1)^{m_{1}+1}$, where $C$ is a closed contour encircling the point $t=1$ counterclockwise and $f$ is analytic within and on $C$. From Cauchy's integral formula, we then find that Eq. (5) becomes

$$
{ }_{D} F_{a}\left[\begin{array}{c}
b_{1}+m_{1}, a_{2}, \cdots, a_{p} ; z \\
b_{1}, b_{2}, \cdots, b_{q}
\end{array}\right]=\frac{D^{m_{1}} f(1)}{\left(b_{1}\right)_{m_{1}}},
$$

$D$ denoting differentiation with respect to $t$. Application of Leibniz's differentiation formula and the wellknown expression for the derivative of a ${ }_{p} F_{q}$ then yields

$$
\begin{aligned}
& { }_{p} F_{q}\left[\begin{array}{c}
b_{1}+m_{1}, a_{2}, \cdots, a_{p} ; z \\
b_{1}, b_{2}, \cdots, b_{q}
\end{array}\right] \\
& =\sum_{j=0}^{m_{1}} z^{j}\left(\begin{array}{c}
m_{1} \\
j
\end{array}\right) \frac{\left(a_{2}\right)_{j} \cdots\left(a_{p}\right)_{j}}{\left(b_{1}\right)_{j} \cdots\left(b_{q}\right)_{j}} \\
& \times_{p-1} F_{q-1}\left[\begin{array}{c}
a_{2}+j, \cdots, a_{p}+j ; z \\
b_{2}+j, \cdots, b_{q}+j
\end{array}\right] .
\end{aligned}
$$

This result can itself be applied to each member of its rhs if $a_{2}=b_{2}+m_{2}$, etc. It is easily seen that the general result (1) is obtained in this way.

A special case $(p=3, q=2)$ of Eq. (8) has been derived by Rösler ${ }^{2}$ from the series representation.

From formula (1) we now derive two summation theorems for $p=q+1, z=1$. These have been given recently by Minton, ${ }^{3}$ the first one, however, under more restrictive conditions than those given below.

To deduce the first theorem, we take $q=p-1=$ $n+1, a_{n+1}=b=b_{n+1}-1, z=1$, and for brevity $a_{n+2}=a$. The hypergeometric functions in the multiple sum of Eq. (1) then become ${ }_{2} F_{1}(1)$ 's, which all exist provided that

$$
\operatorname{Re}(-a)>m_{1}+\cdots+m_{n}-1 .
$$

By Gauss' summation theorem we then get, after some rearrangements,

$$
\begin{array}{r}
{ }_{n+2} F_{n+1}\left(b_{1}+m_{1}, \cdots, b_{n}+m_{n}, b, a ;\right. \\
\left.b_{1}, \cdots, b_{n}, b+1 ; 1\right) \\
=\frac{\Gamma(b+1) \Gamma(1-a)}{\Gamma(b+1-a)} \sum_{j_{1}=0}^{m_{1}} \cdots \sum_{j_{n}=0}^{m_{n}} B_{n}\left(j_{1}, \cdots, j_{n}\right),
\end{array}
$$

where

$$
B_{n}\left(j_{1}, \cdots, j_{n}\right)=\frac{(b+1)_{J_{n}}}{(a)_{J_{n}}}(-1)^{J_{n}} A\left(j_{1}, \cdots, j_{n}\right) \text {. }
$$

Next, the definition (3) is applied, the binomial coefficients being written in the form $(-1)^{j}(-m)_{j} / j$ !; this leads to

$$
\begin{aligned}
B_{n}\left(j_{1}, \cdots, j_{n}\right) & =B_{n-1}\left(j_{1}, \cdots, j_{n-1}\right) \\
\times & \times \frac{\left(b_{n}+m_{n}\right)_{J_{n-1}}}{\left(b_{n}\right)_{J_{n-1}}} \frac{\left(-m_{n}\right)_{j_{n}}\left(b+J_{n-1}\right)_{j_{n}}}{j_{n} !\left(b_{n}+J_{n-1}\right)_{j_{n}}} .
\end{aligned}
$$

The terms containing $j_{n}$ obviously constitute a terminating ${ }_{2} F_{1}(1)$, which is summed by Gauss' theorem. After some rearrangements we obtain (summation limits understood)

$$
\begin{aligned}
\sum_{j_{1}, \cdots, j_{n}} B_{n}\left(j_{1}, \cdots, j_{n}\right) & \\
& =\frac{\left(b_{n}+m_{n}\right)_{-b}}{\left(b_{n}\right)_{-b}} \sum_{j_{1}, \cdots, j_{n-1}} B_{n-1}\left(j_{1}, \cdots, j_{n-1}\right) .
\end{aligned}
$$

Repeating this procedure, we finally arrive at Minton's first theorem,

$$
\begin{aligned}
&{ }_{n+2} F_{n+1}\left(b_{1}\right.+m_{1}, \cdots, b_{n}+m_{n}, b, a ; \\
&\left.b_{1}, \cdots, b_{n}, b+1 ; 1\right) \\
&=\frac{\Gamma(b+1) \Gamma(1-a)}{\Gamma(b+1-a)} \prod_{k=1}^{n} \frac{\left(b_{k}+m_{k}\right)_{-b}}{\left(b_{k}\right)_{-b}},(10)
\end{aligned}
$$

valid under the condition (9), i.e., if the lhs of (10) exists at all. In Minton's proof, ${ }^{3} a$ was required to be negative integral.

The particular case $n=1$ of Eq. (10) was obtained by Mitra $^{4}$ by series manipulations.

The second summation theorem may be deduced from the first ${ }^{3}$ by letting $b \rightarrow \infty$. It may, however, also be deduced directly from Eq. (1) by taking $q=$ $p-1=n, a_{n+1}=-\left(m_{1}+\cdots+m_{n}\right)$, and $z \rightarrow 1$. The hypergeometric functions in the multiple sum of Eq. (1) then reduce to power functions $(1-z)^{h}$, where $h=-a_{n+1}-J_{n}$ and $h \geq 0$ for all terms. When $z \rightarrow 1$, all terms of the multiple sum will thus tend to zero, except the one for which $-a_{n+1}=J_{n}$, i.e., $j_{i}=m_{i}, i=1,2, \cdots, n$; the limit of this term is $A\left(m_{1}, \cdots, m_{n}\right)$. After some reductions we find the summation formula

$$
\begin{array}{r}
{ }_{n+1} F_{n}\left(b_{1}+m_{1}, \cdots, b_{n}+m_{n},-\left(m_{1}+\cdots+m_{n}\right) ;\right. \\
\left.b_{1}, \cdots, b_{n} ; 1\right) \\
=\frac{(-1)^{m_{1}+\cdots+m_{n}}\left(m_{1}+\cdots+m_{n}\right) !}{\left(b_{1}\right)_{m_{1}} \cdots\left(b_{n}\right)_{m_{n}}}, \quad(11)
\end{array}
$$

which is easily transformed to the form given by Minton. ${ }^{3}$

It may be of interest to compare Eq. (11) with the special case of (10) obtained by taking $b_{n}=b, m_{n}=1$ and then replacing $n-1$ by $n$, viz.,

$$
\begin{gathered}
{ }_{n+1} F_{n}\left(b_{1}+m_{1}, \cdots, b_{n}+m_{n}, a ; b_{1}, \cdots, b_{n} ; 1\right)=0, \\
\operatorname{Re}(-a)>m_{1}+\cdots+m_{n} .
\end{gathered}
$$

${ }^{1}$ A. Erdélyi, Quart. J. Math. Oxford Ser. 8, 267 (1937).

R. Rösler, Z. Angew. Math. Mech. 43, 433 (1963).

3 B. M. Minton, J. Math. Phys. 11, 1375 (1970).

${ }^{4}$ S. C. Mitra, J. Indian Math. Soc. (N.S.) 6, 84 (1942). 